\title{
Fuzzy-based Description of Computational Complexity of Central Nervous Systems
}

\author{
Piotr Prokopowicz, Dariusz Mikołajewski, Krzysztof Tyburek, and Piotr Kotlarz \\ Institute of Computer Science, Kazimierz, Wielki University, Bydgoszcz, Poland
}

https://doi.org/10.26636/jtit.2020.145620

\begin{abstract}
Computational intelligence algorithms are currently capable of dealing with simple cognitive processes, but still remain inefficient compared with the human brain's ability to learn from few exemplars or to analyze problems that have not been defined in an explicit manner. Generalization and decision-making processes typically require an uncertainty model that is applied to the decision options while relying on the probability approach. Thus, models of such cognitive functions usually interact with reinforcement-based learning to simplify complex problems. Decision-makers are needed to choose from the decision options that are available, in order to ensure that the decision-makers' choices are rational. They maximize the subjective overall utility expected, given by the outcomes in different states and weighted with subjective beliefs about the occurrence of those states. Beliefs are captured by probabilities and new information is incorporated using the Bayes' law. Fuzzy-based models described in this paper propose a different - they may serve as a point of departure for a family of novel methods enabling more effective and neurobiologically reliable brain simulation that is based on fuzzy logic techniques and that turns out to be useful in both basic and applied sciences. The approach presented provides a valuable insight into understanding the aforementioned processes, doing that in a descriptive, fuzzy-based manner, without presenting a complex analysis.
\end{abstract}

Keywords-cognitive deficit, cognitive function, computational simulation, fuzzy descriptors.

\section{Introduction}

One of the biggest challenges faced by contemporary science is concerned with the problem of how external stimuli are gathered, integrated, analyzed and interpreted within the human brain's neural connections, and the manner in which they are then reflected by human behavior (even as a simple response to stimuli). Scientists continuously search for effective algorithms allowing to code/decode information to/from neural tissue. The so-called human connectome may reflect some of the aforementioned information, also that regarding memory and feelings. Brain mapping (reflected in the connectome) still remains the very first step in the process of obtaining the knowledge required. There is a need for a deeper understanding of built-in memories and feelings. The scale of "thought" processes may be much larger than that of processes taking place within neural networks only. Even a simple analysis of a sentence requires more than activity of the neural networks only (that is similar in many healthy people). It also calls for emotional meaning, social context and many other aspects, thus requiring an advanced and sophisticated analysis of numerous diverse factors that are not always selfexplanatory. Hence, there may be a systematic association between sentence meaning and neural network activation features. Experiments such as "Initiative 2015" may allow to copy the structure of a human brain into a computational avatar.

Rapid biological research developments that continue to be observed since the 1990s have brought about a considerably increase in the amount of biological data of various types. The ability to perform a computational analysis of the aforementioned data sets has given rise to a belief that it will be possible to reflect the full understanding of nature and functionalities of living organisms, and especially of the human central nervous system (CNS). Computational models are regarded as scientific tools linking the commonly known hypotheses and outcomes of experimental studies. Such an approach allows to analyze, in great detail, the building blocks of the human body. Unfortunately, not that much attention is paid to interactions between them, despite the fact that we are aware that the aforementioned interactions may play crucial roles, for instance in more cognitive functions. Such building blocks of the human brain are usually interconnected by a very dense and complex network of bidirectional interactions. The assumption that the structure of this network may determine some basic features of human brain's functioning may be true. Despite the development of novel techniques, such as functional magnetic resonance imaging (fMRI) or positron emission tomography (PET), the current state of our knowledge may be strongly limited by the restricted scope of analyses performed using the existing tools which are poorly suited to cope with the interaction networks referred to above. There is a need for further adaptation of computational methods to the specific nature of neurobiological systems that are dealt with by biology of neurosystems [1]. 


\subsection{Scope of the Paper}

This article presents a short description of computational complexity levels and uncertainty of information, based on an example of degenerative changes and their neural correlates. The concept of their computational simulation is described as well, and the associated discussion is presented. First, a review of current knowledge concerning the investigated group of disorders and computational approaches to associated neurodegenerative changes analysis is presented, as it may help develop new fuzzy-based therapeutic strategies tailored to the profile of a specific patient. Next, the paper proposes a consistent computational method for describing and modeling such a complexity. The authors aim to offer a coherent concept focusing on cost efficiency. The precise methods that are available currently are expensive and time-consuming. Furthermore, connectivity computed with the use of different methods may differ significantly. Despite the efforts taken, results obtained with the use of diffusion tensors, spherical deconvolution, ball-and-stick models, persistent angular structures, etc., may not be the same as those of deterministic and probabilistic tractography algorithms relying on human datasets. This may be caused by the fact that connectivity may be presented in many ways, e.g. as matrices, connectograms. Fiber crossing models are able to reveal connections between more brain areas than a simple tensor model, and probabilistic approaches show more connected regions but lower connectivity values than deterministic methods.

Section 2 presents a review of current state of knowledge in the field that this paper is concerned with. Section 3 introduces computational complexity of brain description, based on the case of Alzheimer's disease. Section 4 presents the fuzzy-based proposal for computations and description brain elements. It is followed by a discussion and, finally, conclusions are presented in Section 6 of the paper.

\section{Computational Complexity}

Generalization and decision-making typically require an uncertainty model related to the decision options and using tools based on the probability theory. Models of such cognitive functions usually interact with reinforcement learning in order to simplify complex problems. Decision-makers are assumed to choose from the available decision options to ensure that their choices are rational. They maximize the subjective, overall utility expected, given by outcomes in different states, weighted with subjective beliefs about the occurrence of those states. Beliefs are captured by probabilities and new information is incorporated using the Bayes law.

Fuzzy-based models propose a different approach - they may give rise to a family of novel methods for a more effective and neurobiologically reliable brain simulation based on fuzzy logic techniques. They may be useful in such complex situations as moral principles guiding social decision-making (the so-called moral strategy), even if different participants spontaneously and consistently employ different moral strategies (guilt aversion, inequity aversion, moral opportunism) in situations in which people adaptively switch between them.

Anatomical connectivity (AC), as an example of structural connectivity, reflects the connectome through synaptic contacts between neighboring neurons or fiber tracks connecting neuron pools in spatially distant brain regions. Functional connectivity (FC) reflects the temporal dependency of neuronal activation patterns of anatomically separated brain regions, i.e. statistical dependencies between distinct and distant regions of information processing neuronal populations. FC refers to interregional synchrony of low-frequency fluctuations, where low denotes frequencies of $v \leq 0.1 \mathrm{~Hz}$. The term synchronize refers to generalized synchronization which is defined through the mapping:

$$
\Psi: S->R \text {, }
$$

where $S$ is the seed phase space, $R$ is the response phase space:

$$
a_{i}(t)=\Psi\left[a_{j}(t)\right],
$$

with some functional $\Psi$ and related seed $a_{s}(t)$ and response $a_{r}(t)$ activities, where $i=s$, and $j=r$ for subsequent states $i, j$ across the spaces mentioned above [2], [3].

There are two basic computational methods determining whether other regions are functionally connected to these seeds based on:

- cross-correlation analysis (CCA) between the BOLD time courses of the seed region and any other brain region, measured by the Pearson correlation coefficient $\rho_{q s}$ such as:

$$
\begin{aligned}
& \rho_{q s}(\tau)=\frac{\sigma_{q s}(\tau)}{\sqrt{\sigma_{q} \cdot \sigma_{s}}}= \\
& =\frac{\left\langle\left(a_{q}(t+\tau)-\left\langle a_{q}(t)\right\rangle\right)\left(a_{s}(t)-\left\langle a_{s}(t)\right\rangle\right)\right\rangle}{\sqrt{\left\langle\left(a_{q}(t+\tau)-\left\langle a_{q}(t)\right\rangle\right)\right\rangle^{2}} \cdot \sqrt{\left.\left\langle a_{s}(t)-\left\langle a_{s}(t)\right\rangle\right)\right\rangle^{2}}},
\end{aligned}
$$

where $\tau$ - predefined time lag $\sigma_{i}$ - variance of regional activity in the query region $(i=q)$ or seed region $(i=s) \sigma_{q s}(\tau)=\left(a_{q}(t+\tau)-a_{(}(t)\right)\left(a_{s}(t)-a_{s}(t)\right)-$ covariance of the fluctuations in neuronal activity in the query and seed regions, respectively,

- coherence in the frequency domain:

$$
H_{q s}(v)=\frac{\left|S_{q s}(v)\right|^{2}}{S_{q, q}(v) \cdot S_{s, s}(v)},
$$

where: $S_{i} j(v)$ is the Fourier cross-spectrum $(i=$ $q, j=s)$ or the Fourier power spectrum $(i=j=q, s)$ of the related covariance functions,

- coherence in the frequency domain [3]:

$$
H_{q s}(v)=\frac{\left|S_{q s}(v)\right|^{2}}{S_{q, q}(v) \cdot S_{s, s}(v)} .
$$

Determination of decomposition and clustering requires the application of one of the following techniques. 
The first one is singular value decomposition (SVD) [3]:

$$
X=U \Sigma V^{T}
$$

where: $X: N \times M$ dimensional matrix of zero mean data with all $M$ functional images concatenated into $M$ column vectors containing $N \cdot M$ pixels each, $U: N \times N$ dimensional matrix of eigen images of the $N \times N$-dimensional correlation matrix, $V: M \times M$ dimensional matrix of eigenvectors of the corresponding kernel matrix $K=X^{T} X, \Sigma$ : rectangular $N \times M$-dimensional matrix containing non-negative, real-valued singular values along its diagonal with only $\min (M, N)$ singular values being different from zero.

The next two methods are principal component analysis (PCA):

$$
X X^{T}=U \Sigma \Sigma^{T} U^{T}=\tilde{U} \tilde{U}^{T},
$$

and independent component analysis (ICA):

$$
X^{T}=M H
$$

where: $X^{T}$ : matrix containing spatial activity distribution in its rows and different observation time points in its columns, $H$ : matrix containing, in its rows, independent spatial activity distributions which are assumed to best characterize the observations, $M$ : containing in its rows the corresponding weights with which each independent component contributes to the observation at any given time point.

The last technique is non-negative matrix and tensor factorization (NMF/NTF) [3].

An alternative approach consists in the application of multivariate auto-regressive models (MVAR) [4], [3]. Effective connectivity (EC) reflects the influence that one neuronal system exerts upon another, thus reflecting causal interactions between activated brain areas, i.e. combines structural and effective connectivity [3].

Structural connectivity has been studied by modeling statistical dependence between the features of cortical regions. The main statistical metrics used include correlations between gray matter thickness. In addition, it has been proposed that partial correlations be used for inferring brain structural connectivity using gray matter volume, instead of the Pearson correlation, since this approach removes the effects of confounding correlations introduced due to canonical dependence between data (Figs. 1-3). Three basic factors play a key role here: methods of parcellation of the cortex (i.e. node selection criteria), threshold selection (i.a. for connection metrics), strength of the relationship between anatomical structure and cognitive function (both in healthy and damaged nervous systems).

Further on in the text, a proprietary approach to the Alzheimer's disease (AD) is proposed. AD is the most important age-related neurodegenerative disorder in developed societies. Prevalence of AD in Europe was estimated at $5.05 \%$. Prevalence in women is higher $(7.13 \%)$ compared to $3.3 \%$ in men, and increases with age. Incidence of $\mathrm{AD}$ in Europe was 11.08 per 1000 person-years. Incidence in women is 13.25 per 1000 person-years men, compared to 7.02 per 1000 person-years in men, and increases with age. (a)

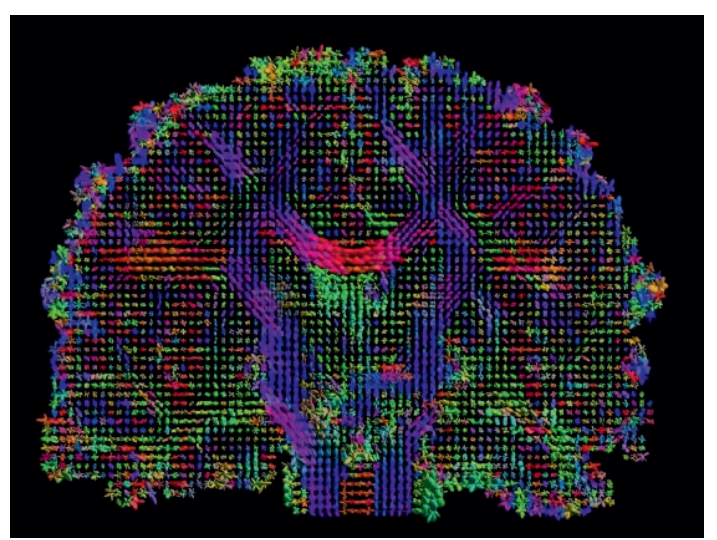

(b)

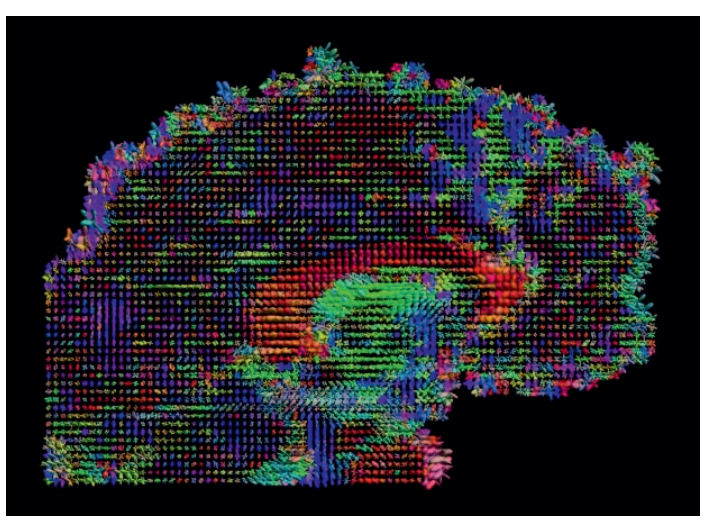

(c)

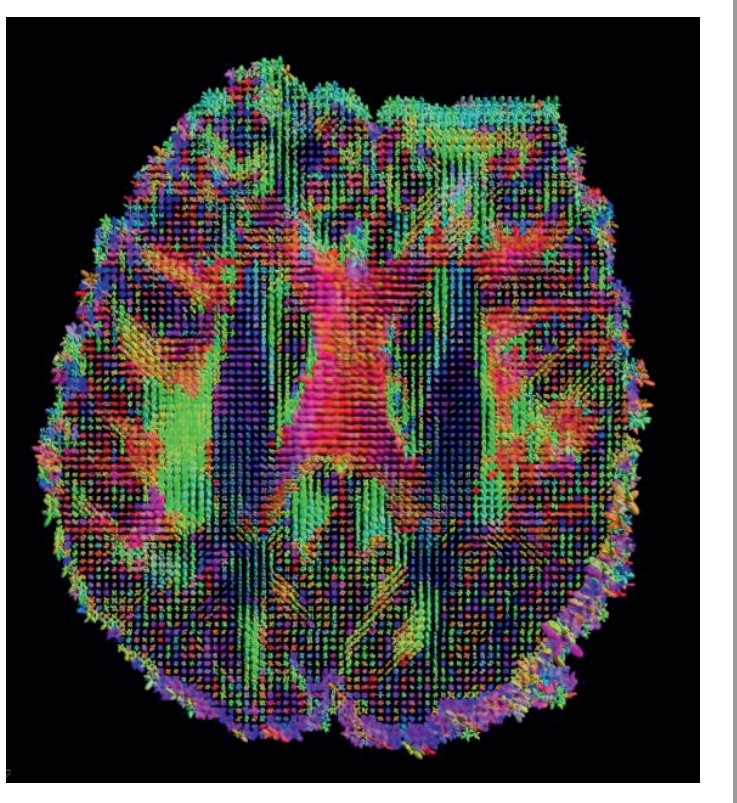

Fig. 1. High-angular resolution diffusion imaging of the human brain: (a) coronal view, (b) sagittal view, (c) axial view; red color describes left-right fibers, green shows anterior-posterior fibers, and blue indicates fibers through the brainstem [5]. (For color pictures see the electronic version of the paper).

Currently, AD diagnosis is based on a subtle cognitive decline in the patient's preclinical health status, detected using neuropsychological tests. Treatment is based on symptomatic therapies, and research enhancing early detection of the disease and preventing pathological changes in the 


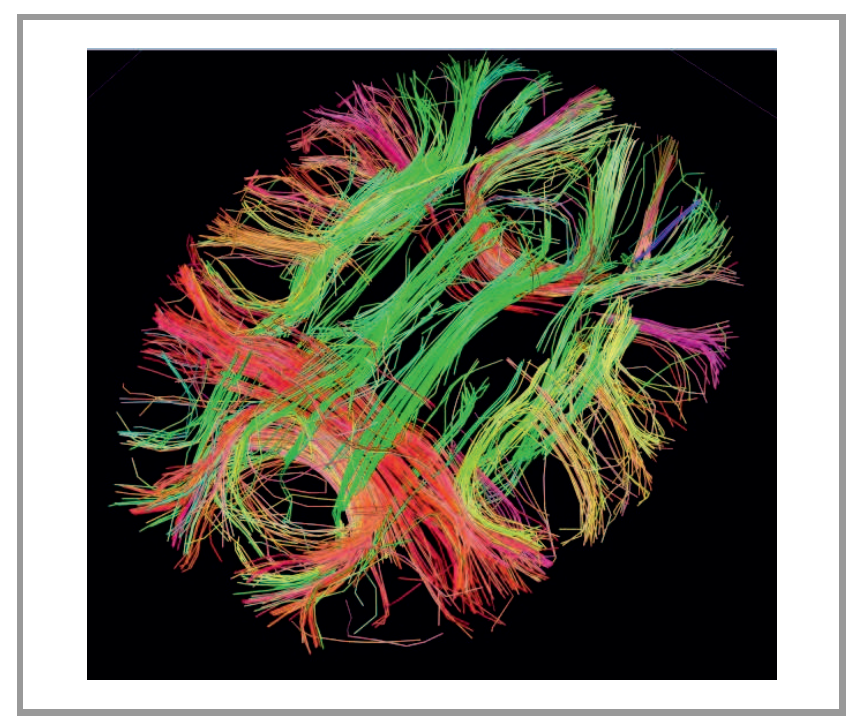

Fig. 2. White matter fiber architecture of the brain; red color shows left-right fibers, green presents anterior-posterior fibers, and blue color illustrates fibers through the brainstem [5].

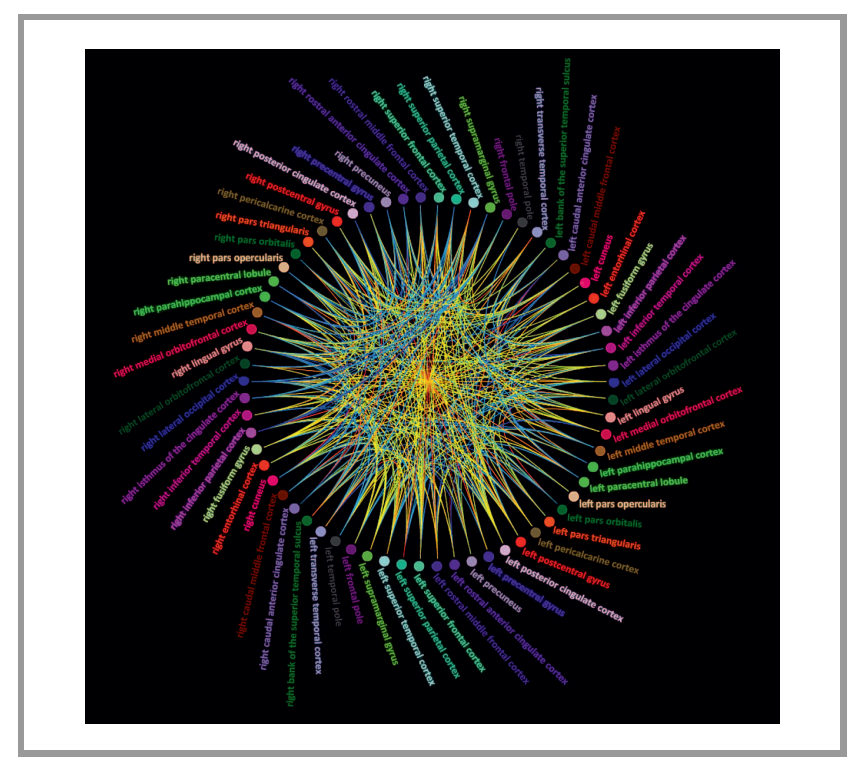

Fig. 3. Architecture of complex interconnections of the brain; warmer colors identify stronger connections and cooler colors identify weaker connections [5].

brain is of key importance. Computational models may significantly increase the understanding of the mechanism underlying the onset of neurodegenerative diseases, and may support development of novel diagnostic and therapeutic methods. Only several studies published over the past decade have been identified line [6]-[12].

\section{Alzheimer Disease Case}

\section{1. aMCI and Late Life Depression as AD Predictors}

$\mathrm{AD}$ is one of the most prevalent neurogenerative brain disorders, affecting an increasing number of people in aging societies of developed and developing countries and creating a number of medical, social and economic challenges. Current research focuses on the following key issues: increasing number of people diagnosed with $\mathrm{AD}$, development of effective therapies and decreasing costs associated with treating and monitoring patients. The diagnostic criteria remain ambiguous, making it difficult to recognize AD symptoms as early as possible. The amnestic form of mild cognitive impairment (aMCI) is regarded as a precursory phase of AD. Even $75-80 \%$ of patients with aMCI finally develop AD [13].

Cognitive functions deficits are often accompanied or preceded by emotional, behavioral and motivational disorders. Late life depression occurs in up to $20 \%$ of patients with $\mathrm{AD}$ and affects about $3.0-4.5 \%$ of adults aged 65 and over [14], [15]. Both aMCI and late life depression are perceived to be risk factors for the onset of dementia in elderly people, being also significant early markers thereof. Elderly people experience health problems that are accompanied by dementia and often also by depression. By taking into account late age depressive disorders in determining the pathogenesis of neurodegenerative processes, another step towards their better understanding may be taken. Research concerning relationships between dementia and depression, their causes, clinical manifestations and treatment is of significant clinical importance.

AD constitutes a dominant form of dementia in Europe (up to $60 \%$ of all cases). The exact percentage share of patients with late life depression converting into aMCI or dementia is still unknown, especially taking into consideration scarce knowledge about the preventive influence of antidepressants in the deterioration of cognitive functions. A close dependency may be observed here: occurrence of dementia impacts the psycho-pathological picture of depression and, vice versa, depression may dramatically worsen the health status of elderly patients. There is no doubt that aMCI patients display more severe cognitive impairments affecting all domains, while late life depression individuals show worse executive function- and memoryrelated results [16]. Unfortunately, no validated markers are capable of precisely predicting the conversion from aMCI to AD. Current clinical diagnosis is based mainly on observation and neuropsychological examination, as well as on AD biomarkers: $\beta$-amyloid in cerebrospinal fluid, use of PET and assessment of neuron damage with the use of tau marking, assessment of brain metabolism in PET and cerebral atrophy in MRI [17].

\subsection{Neural Correlates of aMCI and Late Life Depression}

Structure and function of the central nervous system (CNS) of patients with (a)MCI, depression and AD differ from those of healthy elderly individuals [18], [19]. However, exact differences within brain structures and functions between these MCI subtypes, depression, and AD have not been studied yet. Neuroimaging as a way of determining the conversion of aMCI patients with apolipoprotein E4 
(ApoE-E4, aMCI-E4 patients) to AD was researched by Chen et al. [20]. Impaired structure and function of the hippocampus, constituting a part of the medial temporal lobe memory system, is regarded as a valuable predictor of progression from aMCI to AD. As it is affected by neuropathological changes at the earliest phase, it may show progressive degeneration associated with progression from aMCI to AD [20]. Increased diagnostic efficiency may show a combination of biomarkers derived from neuroimaging (especially MRI), ApoE, and cognitive scores [21].

Hippocampus, posterior cingulate cortex and corpus callosum are currently the key areas of studies focusing on aMCI and AD that are based on the use of structural MRI (sMRI), functional MRI (fMRI), and diffusion tensor imaging (DTI). The neuroimaging abnormalities observed during the progression of aMCI to $\mathrm{AD}$ may affect, primarily, posterior brain regions and may gradually progress to the anterior areas. Current results of the aforementioned studies, however, are insufficient to build effective predictive models that could be applied in screening and diagnosing aMCI and AD. As far as the assessment of the specific risk of conversion from aMCI to $\mathrm{AD}$ is concerned, the Brain Age Gap Estimation (BrainAGE) method, determining the impact of ApoE genotype on structural brain aging, proves to be an accurate tool, even if information on the patient's ApoE status is missing [21]. A multi-tool approach (sMRI, fMRI, DTI) has been adopted by Yu et al. in [23] to analyze neuroimages of brain in patients with aMCI and vascular MCI (VaMCI). Microstructural alterations revealed by multimodal MRIs, including sMRI, resting-state functional MRI and DTI, and accompanied by neuropsychological changes, were observed in [24]. The results of recent EEG-based studies focusing on aMCI, late life depression and $\mathrm{AD}$, as well as on the diagnostic value of spectral analysis of EEG (at rest) in the process of differentiating aMCI in routine clinical practice, are questioned. Analysis of synchronicity of bioelectrical activity of the brain may improve the situation. Combination of neurophysiological and neuroimaging methods may provide much better outcomes.

\subsection{Computational Concepts of aMCI Simulation}

The correlation between cognitive states and network characteristics is rather clear, but remains multi-factorial. From the computational modeling point of view, $\mathrm{AD}$ has been previously linked with the concept of underlying pathological brain network disorganization and less efficient information processing.

The current approach has not provided explanations for pathological alterations of brain network functions during the preclinical phase of aMCI yet. The following types of functional disorganization was shown:

- functional disorganization correlated with the participant's generic cognitive status in healthy people, in patients with aMCI and with mild dementia,
- "small world" brain architecture identified in aMCI patients relative to patients with mild dementia,

- significant reduction in brain architecture in both aMCI and MD patients, relative to the group of healthy controls.

Pathological changes in the organization of brain networks are caused mainly by deficient local information processing (lower local clustering, reduced strength of functional hubs), despite some compensatory phenomena observed and having the form of the formation of additional hubs in left frontal and parietal regions. The aforementioned changes resulted in declined functional organization of the network, even during the prodromal/preclinical phase [23]. On the other hand, induction of neuroplasticity of the mature human brain by combining cognitive and physical activity (e.g. dancing) leads to the prevention of dementia. Traditional Greek dances may improve optimal network performance (small-world properties, local network changes causing better information flow and functional reorganization of the network nodes) [25]. Moreover, there may be diverse computational mechanisms of aMCI, since various aMCI clinical sub-types have been identified as being of the single domain (SD) or multi-domain (MD) variety, resulting in the heterogeneous syndrome with different probabilities of progression from aMCI to AD. Abnormalities underlying MD-aMCI distributed across temporal, frontal, and parietal cortices showed that MD-aMCI is a transition stage between SD- aMCI and AD [26].

The brain network in aMCI patients displayed a disconnection syndrome and a loss of small world architecture. The aforementioned features may be influenced by numerous factors - see e.g. deterioration of the cognitive state in diabetic patients, reflecting a less optimal network organization [27]. Cortical sources small worlds net of resting EEG in eyes-closed/open differs in aMCI and AD patients in comparison to healthy subjects. The aMCI small world architecture presents midway properties between AD and healthy subjects, but in the low frequencies (delta and theta bands), the aMCI group presents a network architecture that is similar to that of healthy individuals. Such results may confirm the hypothesis stating that aMCI constitutes an intermediate step in the progression of AD [28].

Small world topology networks constructed by cortical mean diffusivity in aMCI and AD are not well known so far. But one may state that patients with aMCI and AD show abnormal small-world properties of cortical diffusivity networks reflected by a higher degree of clustering and longer path lengths, i.e. less optimal topological organization. According to the best current knowledge, changes of cortical diffusivity in topological network organization, the average complexity of connections and the disrupted hub regions in aMCI may be useful in identifying patients in the prodromal stage of $\mathrm{AD}$, as they reflect microstructural neurodegeneration [29]. Network construction optimization problems may be a key for a deeper understanding of the 
complexity of the transition between healthy, aMCI, and AD states [30]-[32].

Our approach may be simpler than a complete solution to the aforementioned problem. The complete solution requires that two areas be converged:

- underlying mathematical model of the partial differential equation (PDE, e.g. Bloch-Torrey PDE), harmonic analysis, and algebraic geometry,

- technology, primarily diffusion magnetic resonance imaging (dMRI).

The aforementioned approach creates an efficient solution to the 3-dimensional imaging problem. It is achieved firstly by the reconstruction of a homogeneous polynomial representing a real-valued function on a sphere, based on dMRI data. Then, these data sets are analyzed by being decomposed into a sum of powers of linear forms. The ultimate result requires combination and mapping of neural fibers, i.e. multiple-fiber reconstruction of the brain map. Multiple compartment transformation of the Bloch-Torrey PDE is used to reduce oscillations in the searched-for solution and simplifies the implementation of the boundary conditions:

$$
\begin{gathered}
\frac{\delta}{\delta t}=-I \gamma f(t) G(r) M(r, t)+\nabla \cdot(D(r) \nabla M(r, t), \\
r \in \bigcup \Omega^{I}
\end{gathered}
$$

and for each compartment $\Omega^{I}$ :

$$
\begin{aligned}
& \frac{\delta}{\delta t} \int_{\Omega^{I}} M v \mathrm{~d} r=-I \gamma f(t) \int_{\Omega^{I}} G M v \mathrm{~d} r \\
& -\int_{\Omega^{I}} D \nabla M \cdot \nabla v \mathrm{~d} r+\int_{\delta \Omega^{I} \cap \Gamma} D \nabla M \cdot n^{l} v \mathrm{~d} s \\
& +\int_{\delta \Omega^{I} \cap \delta C} D \nabla M \cdot n^{l} v \mathrm{~d} s .
\end{aligned}
$$

Then, the similarity, weak form of the steady-state Laplace PDE is:

$$
\begin{aligned}
& \int_{\Omega^{I}} D \nabla W \cdot \nabla v \mathrm{~d} r-\int_{\delta \Omega^{I} \cap \Gamma} D \nabla W \cdot n^{l} v \mathrm{~d} s \\
& +\int_{\delta \Omega^{I} \cap \delta C} D \nabla W \cdot n^{l} v \mathrm{~d} s=0,
\end{aligned}
$$

where: $\gamma=2.67513 \cdot 10^{8} \mathrm{rads}^{-1} T^{-1}, D(r)-$ intrinsic diffusion tensor, $n^{l}$ - outward pointing normal, $v-$ test function, $M(r, t)$ - magnetization, function of position $r$ and time $t$, depends on diffusion gradient vector $g$ and time profile $f(t)$ [33].

EniCS finite elements platform and the Salome mesh generator may be applied to solve the aforementioned problems numerically.

As far as less efficient information processing is concerned, brain network dysfunction analyzed at the systemic level may be a key issue: aMCI with AD showed abnormali- ties both in regional brain activation and large-scale brain networks:

- aMCI patients show hypoactivation in default, visual, and frontoparietal networks, relative to healthy controls,

- AD patients show hypoactivation in default, visual, and ventral attention networks, relative to healthy controls,

- both group of patients (aMCI and AD) show hyperactivation in default, frontoparietal, ventral attention, and somatomotor networks, relative to healthy controls,

- MCI and AD present different pathological changes, sharing even similar compensatory large-scale networks relied upon in fulfilling cognitive tasks [32].

The response time (RT) necessary for safe, appropriate, and efficient environmental interaction required in real life is normally distributed for cognitively healthy older adults, but not for the aMCI group [22]. A high degree of intraindividual variability (IIV) has been observed, and results reveal a dichotomy between the preservation of RT along with aging, a phenomenon that is probably strongly related to the research methodology used. Current approaches are based on simple clinical tests and scales - there is a lack of a widely accepted integrated computational approach. The first of the aforementioned approaches to brain network disorganization gives a detailed picture of brain connections, but not so of brain mechanisms, especially mechanisms of a higher level of abstraction associated with memory, reasoning and decision-making processes. The other approach (less efficient information processing) shows the behavior and, partly, the associated mechanisms, but requires that the computational description rules reflecting its function be determined.

\section{Fuzzy-based Approach}

Our approach to fuzzy-based computational analysis of brain processes has already been described before [34], [35]. We developed a novel computational tool: hierarchical fuzzy systems based on ordered fuzzy numbers, i.e. the direction of the calculation/operation has its own meaning [36], [37]. Thus, the theory of fuzzy sets offers help in defining formal models in situations in which only a linguistic description is possible. It may be easily adapted to reflect the "fuzziness" of neurodegenerative changes associated with AD and aMCI. A fuzzy description of signal transformation performed through the complex spatial properties of brain networks, from optimization of network structure thanks to long time developmental and learning processes, toward a non-optimized or even distorted connectome due to neurodegenerative processes, and with time complexity of the step-by-step transition between healthy brain, through aMCI, toward AD, 
allows for more precise control and prediction. The modular fuzzy approach proposed better reflects various speeds and other properties of disturbed simultaneous hierarchical data processing, as well as co-occurrence of numerous signals. The models are more flexible and simpler to adapt. Thanks to the aforementioned properties, lowcost less time-consuming simulations, a considerably higher number of variables may be tested, enabling to reflect IIV. Intuitive information provided by experienced brain modeling scientists may be represented in a simpler manner, with growing and decreasing dependencies [38]. Invalidly synchronized (or non-synchronized) structures may be described, for instance by weaker-than-usual co-occurrence of excitation/inhibition, making them hard to detect. Such an approach may help reflect structural connectivity (SC) as a part of functional connectivity (FC).

The aforementioned approach may introduce new quality to various cognitive changes and their differential diagnosis. Changes in the patient's nervous networks may take place much earlier than visible symptoms and diagnostic outcomes may be identified. Therefore, the proposed approach simultaneously creates a need for novel brain state visualization tools. Furthermore, the proposed fuzzy-based approach allows for more advanced OFN-based brain function trend modeling. Such an approach may offer an increased predictive value based on current and new knowledge gathered.

A proper choice of fuzzification and defuzzification method exerts a key impact on the correctness and effectiveness of the entire model, including accumulation of losing bits of information (see [39]). Our proposal processes a complex functionality that is expressed linguistically by a fuzzy system, without fuzzification and defuzzification stages used as a fuzzy inference block (FIB). Moreover, its conceptual basis is enhanced by cascade-based modeling of complex relations, described linguistically. Every FIB may represent one agent from the multiagent architecture. Despite the high degree of complexity of the model, we can still describe relations at various stages linguistically. Multi-input and multi-output semi-cascades of FIBs may simultaneously reflect various conditions and co-operation states of diverse brain networks, with the required scalability. Hence, it seems that the small world network is widely accepted as a scalable intrinsic miniature of the nervous system.

The maximum value of the reward function $R$ guarantees that the best change may be found in a given step. The reward function changes dynamically during the time steps. Therefore, better choice is improving other parameters e.g. learning rate coefficient. The change of the weight between $n_{i}$ and $n_{j}$ is calculated as:

$$
\frac{d w_{i j}}{d t}=a R(t) e_{i j}(t)
$$

where: $a$ - learning rate, $R$ - reward function, $e_{i j}$ - eligibility trace between $n_{i}$ and $n_{j}$ neurons from the two adjacent layers.
In our approach, we focus inter alia on modeling the learning rate with OFN, and on trend analysis. Such an approach may reflect the stochastic behavior of simple, reliable, very fast, low-power computing devices embedded in recursive brain-like architectures.

\section{Discussion}

Computational neuroscience is developing rapidly and provides a variety of modern tools for analyzing functional interactions of the brain and for detecting pathological deviations caused by neurodegeneration. Identification of functional and neural markers enabling to diagnose adverse cognitive changes associated with MCI and depression, and leading to an early diagnosis of $\mathrm{AD}$, is a key objective of modern neuroscience and geriatrics. Unfortunately, there is lack of comparable reviews. What more there is hard to cover all possible issues and factors in a single study.

The point of view of the authors formulates a concept that is still at an early stage of development and needs further discussions. It aims at ensuring lower computational complexity, as well as wide acceptance and availability, but still suffers from many limitations that are mentioned below. Further tests and numerical results, including compartmental studies, will be presented in the future. However, an advanced approach based on computational intelligence seems to constitute another breakthrough in brain function modeling.

Limitations of the proposed concept are twofold: they result from the complexity of the brain (and lack of its direct modeling) and from the characteristics of the proposed concept. The diversified location of brain changes, the level neural correlations between aMCI and depression, the connection between distant areas of CNS, diverse clinical subtypes, and a few clear "cause-effect" relationships are the main challenges that need to be faced while conducting research. There is a need for novel approaches which meet all of the abovementioned criteria, simultaneously being more complex and precise, offering enough explanatory and predictive power, as well as allowing for further development of computational models of neurologic diseases and deficits in elderly patients [40], [41], [42], [43].

Computer tools allow to collect and analyze (with the use of artificial intelligence) significant amounts of information. Diverse combinations, such as AD only, AD and coexisting depression, $\mathrm{AD}$ with aMCI, AD with aMCI and associated depression, or $\mathrm{AD}$ with depression, may be identified in an easier manner. The area and progress of pathological CNS processes should be defined by means of a certain boundary point, thus enabling them to be distinguished precisely.

A comprehensive patient diagnosis is needed to get a clear understanding of the impact of various areas, levels and severities of underlying neurodegenerative changes. The main advantages will have the form of a deeper understanding of dementia's development pathomechanisms. The ulti- 
mate result will be a flexible, multidimensional diagnostic procedure, enabling to early detect pre-AD effects and to apply a suitable treatment wherever possible.

\subsection{Directions for Further Research}

Future research should focus primarily on developing a software tool, but there are many other issues that may contribute to a rapid increase in the potential of the proposed approach. A fuzzy system may be suitable for shape the control signal and for developing much more advanced control structures. Fuzzy logic and model predictive control methodologies may create a novel multi-parametric programming (MPT) approach. Advanced BCI applications require real time solutions, but brain subsystems simulation requires only low-cost post-hoc operation. Therefore, the use of the fuzzy MPT technique may be an efficient and a relatively simple and cheap solution [44].

Despite the great expressive power of fuzzy systems, they also suffer from specific limitations in terms of modeling neurobiological systems. Determination of exact values of some parameters and of the associated structures is not always possible. Therefore, it is difficult to exactly correspond to some quantitative properties of the modeled system. Even a formal analysis of graph-based models, such as Petri nets (structure of a directed bipartite graph), may be general. We should be also aware that the lead time of neurobiological responses exerts a significant influence on the overall behavior of the human body and on the modeled system. Hence, Petri nets may enable, with time, to perform detailed and accurate modeling and analysis of the modeled system [1].

There are also other concepts that should be taken into consideration in the course of future studies, such as granular computing [45], [46] or flexible and reliable fuzzy logicbased decision support systems [47].

\section{Conclusions}

In this paper, the concept of a fuzzy logic-assisted decision support system (DSS) has been introduced. Thanks to FL, the proposed solution provides a valuable insight into the understanding of the aforementioned processes, relying on descriptive, fuzzy-based methods, without the need to perform a complex analysis. Early and precise identification of patients with aMCI, late life depression or $\mathrm{AD}$ is of great significance for introducing successful prevention and intervention programs. Future studies on aMCI and late life depression should cover computational models and ought to provide stronger evidence allowing to adopt a novel approach to prevention, diagnosis and treatment of AD. Such an approach may fill the existing gap between theoretical hypotheses and experimental studies. The majority of components of the aforementioned mechanisms and associated treatment strategies still need to be confirmed by future studies.

\section{References}

[1] J. Olszak, M. Radom, and P. Formanowicz, "Some aspects of modeling and analysis of complex biological systems using time Petri nets", Bull. of the Polish Acad. of Sci.: Tech. Sci., vol. 66, no. 1, pp. 67-78, 2018 (DOI: 10.24425/119060).

[2] D. He, Z. Zheng, and L. Stone, "Detecting generalized synchrony: An improved approach", Phys. Rev. E, vol. 67, no. 2, 026223, 2003 (DOI: 10.1103/PhysRevE.67.026223).

[3] E. W. Lang, A.M. Tomä, I. R. Keck, J. M. Gêrriz-Sçez, and C. G. Puntonet, "Brain connectivity analysis: A short survey", Comput. Intell. and Neurosci., vol. 2012, article ID 412512, pp. 1-21, 2012 (DOI: 10.1155/2012/412512).

[4] M. Krumin and S. Shoham, "Multivariate autoregressive modeling and granger causality analysis of multiple spike trains", Comput. Intell. and Neurosci., vol. 2010, article ID 752428, pp. 1-9, 2010 (DOI: 10.1155/2010/752428).

[5] Human Connectome Project [Online]. Available: www.humanconnectomeproject.org

[6] W. de Haan, E. C. W. van Straaten, A. A. Gouw, and C. J. Stam, "Altering neuronal excitability to preserve network connectivity in a computational model of Alzheimer's disease", PLOS Comput. Biol., vol. 13, no. 9, e1005707, 2017 (DOI: 10.1371/journal.pcbi.1005707).

[7] H. Niu, I. Álvarez-Álvarez, F. Guillén-Grima, and I. AguinagaOntoso, "Prevalencia e incidencia de la enfermedad de Alzheimer en Europa: metaanálisis", Neurología, vol. 32, no. 8, pp. 523-532, 2017 (DOI: 10.1016/j.nrl.2016.02.016).

[8] J. R. Petrella, W. Hao, A. Rao, and P. M. Doraiswamy, "Computational causal modeling of the dynamic biomarker cascade in Alzheimer's disease", Comput. and Mathem. Methods in Med., vol. 2019, pp. 1-8, 2019 (DOI: 10.1155/2019/6216530).

[9] P. D. Roberts, A. Spiros, and H. Geerts, "Simulations of symptomatic treatments for Alzheimer's disease: Computational analysis of pathology and mechanisms of drug action", Alzheimer's Res. \& Ther., vol. 4, no. 6, 2012 (DOI: 10.1186/alzrt153).

[10] S. J. B. Vos et al., "Prevalence and prognosis of Alzheimer's disease at the mild cognitive impairment stage", Brain, vol. 138, no. 5, pp. 1327-1338, 2015 (DOI: 10.1093/brain/awv029).

[11] J. Weller and A. Budson, "Current understanding of Alzheimer's disease diagnosis and treatment", F1000Res., vol. 7, 1161, 2018 (DOI: 10.12688/f1000research.14506.1).

[12] F. Zhu et al., "COMPASS: A computational model to predict changes in MMSE scores 24-months after initial assessment of Alzheimer's disease", Scient. Rep., vol. 6, no. 1, 2016 (DOI: 10.1038/srep34567).

[13] Z. Hu, L. Wu, J. Jia, and Y. Han, "Advances in longitudinal studies of amnestic mild cognitive impairment and Alzheimer's disease based on multi-modal MRI techniques", Neurosci. Bull., vol. 30, no. 2, pp. 198-206, 2014 (DOI: 10.1007/s12264-013-1407-y).

[14] V. Valkanova, K. P. Ebmeier, and C. Allan, "Depression is linked to dementia in older adults", Practitioner, vol. 261, no. 1800, pp. 11-15, 2017 [Online]. Available: https://pubmed.ncbi.nlm.nih.gov/29023080/

[15] B. S. Diniz, M. A. Butters, S. M. Albert, M. A. Dew, and C. F. Reynolds, "Late-life depression and risk of vascular dementia and Alzheimer's disease: systematic review and meta-analysis of community-based cohort studies", British J. of Psychiatry, vol. 202, no. 5, pp. 329-335, 2013 (DOI: 10.1192/bjp.bp.112.118307).

[16] W. Liao et al., "The characteristic of cognitive dysfunction in remitted late life depression and amnestic mild cognitive impairment", Psychiatry Res., vol. 251, pp. 168-175, 2017

(DOI: 10.1016/j.psychres.2017.01.024).

[17] G. M. McKhann et al., "The diagnosis of dementia due to Alzheimer's disease: Recommendations from the national institute on aging-Alzheimer's association workgroups on diagnostic guidelines for Alzheimer's disease". Alzheimer's \& Dement., vol. 7, no. 3, pp. 263-269, 2011 (DOI: 10.1016/j.jalz.2011.03.005).

[18] P. Yu et al., Enriching amnestic mild cognitive impairment populations for clinical trials: optimal combination of biomarkers to predict conversion to dementia", J. of Alzheimer's Dis., vol. 32, no. 2 , pp. 373-385, 2012 (DOI: 10.3233/JAD-2012-120832). 
[19] Y. Chow et al., "Limbic brain structures and burnout-A systematic review", Adv. in Medical Sci., vol. 63, no. 1, pp. 192-198 2018 (DOI: 10.1016/j.advms.2017.11.004).

[20] G. Q. Chen et al., "Neuroimaging basis in the conversion of aMCI patients with APOE-e4 to AD: study protocol of a prospective diagnostic trial", BMC Neurol., vol. 16, no. 1, 2016 (DOI: 10.1186/s12883-016-0587-2).

[21] J. Chen, Z. Zhang, and S. Li, "Can multi-modal neuroimaging evidence from hippocampus provide biomarkers for the progression of amnestic mild cognitive impairment?", Neurosci. Bull., vol. 31, no. 1, pp. 128-140 2015 (DOI: 10.1007/s12264-014-1490-8).

[22] J. Haworth, M. Phillips, M. Newson, P. J. Rogers, A. Torrens-Burton, and A. Tales, "Measuring information processing speed in mild cognitive impairment: clinical versus research dichotomy", J. of Alzheimer's Dis., vol. 51, no. 1, pp. 263-275, 2016 (DOI: 10.3233/JAD-150791).

[23] Y. Yu, W. Zhao, S. Li, and C. Yin, MRI-based comparative study of different mild cognitive impairment subtypes: protocol for an observational case-control study", BMJ Open, vol. 7, no. 3, 2017 (DOI:10.1136/bmjopen-2016-013432).

[24] L. C. Lowe, C. Gaser, and K. Franke, "The effect of the apoe genotype on individual brainage in normal aging, mild cognitive impairment, and Alzheimer's disease", PLOS ONE, vol. 11, no. 7, e0157514, pp. 1-25, 2016 (DOI: 10.1371/journal.pone.0157514).

[25] C. A. Frantzidis et al., "Functional disorganization of small-world brain networks in mild Alzheimer's disease and amnestic mild cognitive impairment: an EEG study using relative wavelet entropy (RWE)", Front. in Aging Neurosci., vol. 6, 2014 (DOI: 10.3389/fnagi.2014.00224)

[26] V. I. Zilidou et al., "Functional re-organization of cortical networks of senior citizens after a 24 -week traditional dance program", Front. in Aging Neurosci., vol. 10, 2018 (DOI: 10.3389/fnagi.2018.00422).

[27] H. J. Li et al., "Toward systems neuroscience in mild cognitive impairment and Alzheimer's disease: A meta-analysis of 75 FMRI studies", Human Brain Mapp., vol. 36, no. 3, pp. 1217-1232, 2015 (DOI: $10.1002 / \mathrm{hbm} .22689$ ).

[28] K. Zeng, Y. Wang, G. Ouyang, Z. Bian, L. Wang, and X. Li, "Complex network analysis of resting state EEG in amnestic mild cognitive impairment patients with type 2 diabetes", Front. in Comput. Neurosci., vol. 9, p. 133, 2015 (DOI: 10.3389/fncom.2015.00133).

[29] F. Miraglia, F. Vecchio, P. Bramanti, and P. M. Rossini, "EEG characteristics in "eyes-open" versus "eyes-closed" conditions: Smallworld network architecture in healthy aging and age-related brain degeneration", Clin. Neurophysiol., vol. 127, no.2, pp. 1261-1268, 2016 (DOI: 10.1016/j.clinph.2015.07.040)

[30] B. Zhang et al., "Characterizing topological patterns in amnestic mild cognitive impairment by quantitative water diffusivity", $J$. of Alzheimer's Dis., vol. 43, no. 2, pp. 687-697, 2014 (DOI: 10.3233/JAD-140882).

[31] J. M. Czerniak, H. Zarzycki, and D. Ewald, "AAO as a new strategy in modeling and simulation of constructional problems optimization", Simul. Modell. Pract. and Theory, vol. 76, pp. 22-33, 2017 (DOI: 10.1016/j.simpat.2017.04.001).

[32] X. Li and Z. J. Zhang, "Neuropsychological and neuroimaging characteristics of amnestic mild cognitive impairment subtypes: A selective overview", CNS Neurosci. \& Ther., vol. 21, no. 10, pp. 776-783, 2015 (DOI: 10.1111/cns.12391).

[33] D. V. Nguyen, J. R. Li, D. Grebenkov, and D. Le Bihan, "A finite elements method to solve the Bloch-Torrey equation applied to diffusion magnetic resonance imaging", J. of Comput. Phys., vol. 263, pp. 283-302, 2014 (DOI: 10.1016/j.jcp.2014.01.009).

[34] D. Zhang et al., "Multimodal classification of Alzheimer's disease and mild cognitive impairment", NeuroImage, vol. 55, no. 3, pp. 856-867, 2011 (DOI: 10.1016/j.neuroimage.2011.01.008).

[35] P. Prokopowicz and D. Mikołajewski, "Fuzzy-based computational simulations of brain functions - preliminary concept", BioAlgorith. and Med-Syst., vol. 12, no. 3, 2016 (DOI: 10.1515/bams-2016-0009).
[36] P. Prokopowicz and D. Ślęzak, "Ordered fuzzy numbers: Sources and intuitions", in Theory and Applications of Ordered Fuzzy Numbers: A Tribute to Professor Witold Kosiński, P. Prokopowicz, J. Czerniak, D. Mikołajewski, Ł. Apiecionek, and D. Ślęzak, Eds. Springer, 2017, pp. 47-56 (DOI: 10.1007/978-3-319-59614-3_3).

[37] P. Prokopowicz and D. Ślęzak, "Ordered fuzzy numbers: Definitions and operations", in Theory and Applications of Ordered Fuzzy Numbers: A Tribute to Professor Witold Kosiński, P. Prokopowicz, J. Czerniak, D. Mikołajewski, Ł. Apiecionek, and D. Ślęzak, Eds. Springer, 2017, pp. 57-79 (DOI: 10.1007/978-3-319-59614-3_4).

[38] P. Prokopowicz, "Processing direction with ordered fuzzy numbers", in Theory and Applications of Ordered Fuzzy Numbers: A Tribute to Professor Witold Kosiński, P. Prokopowicz, J. Czerniak, D. Mikołajewski, Ł. Apiecionek, and D. Ślęzak, Eds. Springer, 2017, pp. 81-98 (DOI: 10.1007/978-3-319-59614-3_5).

[39] P. Prokopowicz, "The use of ordered fuzzy numbers for modelling changes in dynamic processes", Inform. Sciences, vol. 470, pp. 1-14, 2019 (DOI: 10.1016/j.ins.2018.08.0)

[40] J. M. Czerniak and H. Zarzycki, "Artificial acari optimization as a new strategy for global optimization of multimodal functions", $J$. of Comput. Sci., vol. 22, pp. 209-227 2017 (DOI: 10.1016/j.jocs.2017.05.028).

[41] J. Masiak, M. Kuspit, W. Surtel, and M. J. Jarosz, "Stress, coping styles and personality tendencies of medical students of urban and rural origin", Ann. of Agricul. and Environ. Med., vol. 21, no. 1, pp. 189-193, 2014 [Online]. Available: http://www.aaem.pl/ pdf-72083-9310?filename=Stress_\%20coping\%20styles\%20and.pdf

[42] G. M. Wojcik et al., "Mapping the human brain in frequency band analysis of brain cortex electroencephalographic activity for selected psychiatric disorders", Front. in Neuroinform., vol. 12, 2018 (DOI: 10.3389/fninf.2018.00073).

[43] G. M. Wojcik et al., "New protocol for quantitative analysis of brain cortex electroencephalographic activity in patients with psychiatric disorders", Front. in Neuroinform., vol. 12, 2018 (DOI: 10.3389/fninf.2018.00027).

[44] P. Prokopowicz and D. Mikołajewski, "OFN-based brain function modeling", in Theory and Applications of Ordered Fuzzy Numbers: A Tribute to Professor Witold Kosiński, P. Prokopowicz, J. Czerniak, D. Mikołajewski, Ł. Apiecionek, and D. Ślęzak, Eds. Springer, 2017, pp. 303-322 (DOI: 10.1007/978-3-319-59614-3_18).

[45] P. Serkies, "A novel predictive fuzzy adaptive controller for a twomass drive system", Bull. of the Polish Acad. of Sci.: Tech. Sci., vol. 66, no. 1, pp. 37-47, 2018 (DOI: 10.24425/119057).

[46] A. Piegat and M. Plucinski, "Fuzzy number division and the multigranularity phenomenon", Bull. of the Polish Acad. of Sci., Tech. Sci., vol. 65 , no. 4 , pp. $497-511,2017$ (DOI: 10.1515/bpasts-2017-0055).

[47] M. Jasiński, P. Majtczak, and A. Malinowski, "Fuzzy logic in decision support system as a simple Human/Internet of Things interface for shunt active power filter", Bull. of the Polish Acad. of Sci., Tech. Sci., vol. 64, no. 4, pp. 877-886, 2016 (DOI: 10.1515/bpasts-2016-0096).

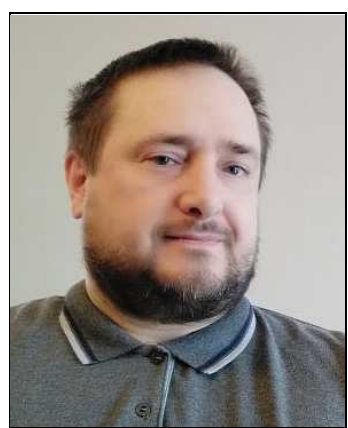

Piotr Prokopowicz received his Ph.D. and completed his habilitation in Computer Science in 2005 and 2020, respectively. He is currently an Associate Professor and the Scientific Council Chairman at the Institute of Computer Science at the Kazimierz Wielki University, Bydgoszcz, Poland. 
Piotr Prokopowicz is an author and co-author of numerous articles in the field of theory and application of artificial intelligence methods, published in journals and conference proceedings. His main research fields include computational intelligence methods, with the focus placed on fuzzy sets and systems. He has been lately working on methods that are sensitive to the direction in which information is flowing.

(iD) https://orcid.org/0000-0002-7223-9008

Email: piotrekp@ukw.edu.pl

Institute of Computer Science

Kazimierz Wielki University

Kopernika 1

85-074 Bydgoszcz, Poland

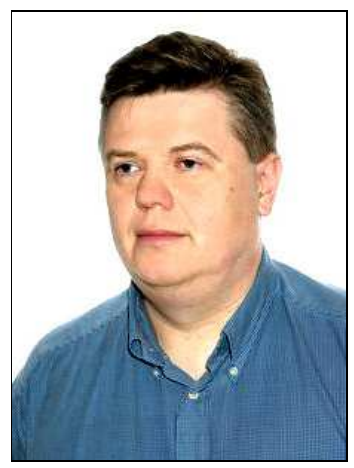

Dariusz Mikołajewski received his Ph.D. from the Institute of Biocybernetics and Biomedical Engineering, Polish Academy of Science in Warsaw, Poland, in 2015. Currently, he is an Associate Professor and the Head of the Department of Teleinformatics and Electronic Devices at the Institute of Computer Science, Kazimierz Wielki University, Bydgoszcz, Poland. He is an author and co-author of 180 scientific publications. His areas of scientific interest include artificial intelligence, applications of IT in medicine, and biocybernetics.

(iD) https://orcid.org/0000-0003-4157-2796

Email: dmikolaj@ukw.edu.pl

Institute of Computer Science

Kazimierz Wielki University

Kopernika 1

85-074 Bydgoszcz, Poland

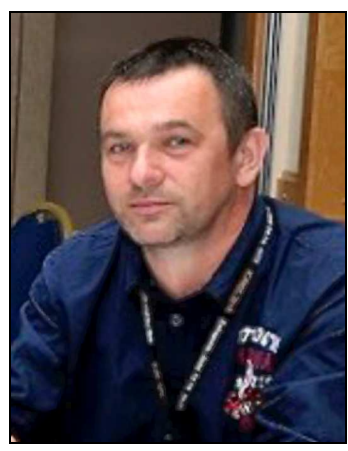

Krzysztof Tyburek received his Ph.D. degree in Computer Science from the Institute of Fundamental Technological Research, Polish Academy of Science, Warsaw, Poland, in 2008. His research focuses on database systems, software engineering and digital signal processing, with a particular emphasis placed on sound recognition features. He has over 20 years of academic experience. Currently, Dr. Tyburek is an Assistant Professor at the Institute of Computer Science, Kazimierz Wielki University, Bydgoszcz, Poland.

(iD) https://orcid.org/0000-0002-1591-4740

Email: krzysztof.tyburek@ukw.edu.pl

Institute of Computer Science

Kazimierz Wielki University

Kopernika 1

85-074 Bydgoszcz, Poland

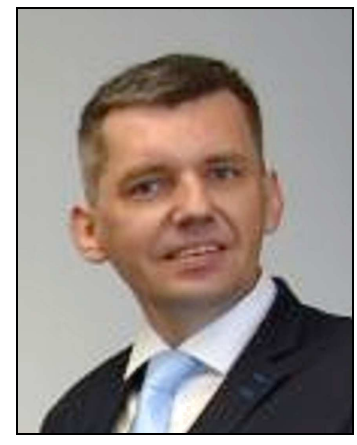

Piotr Kotlarz received his Ph.D. degree in Computer Science from the Institute of Fundamental Technological Research, Polish Academy of Sciences. $\mathrm{He}$ is currently working as an Academic Teacher at Institute of Computer Science, Kazimierz Wielki University. His areas of interest include robotics, cryptography, computer networks and artificial intelligence.

(iD) https://orcid.org/0000-0001-5004-2928

E-mail: piotrk@ukw.edu.pl

Institute of Computer Science

Kazimierz Wielki University

Kopernika 1

85-074 Bydgoszcz, Poland 